\title{
Método Alternativo de Zoneamento Agroclimático do Milho para o Estado de Alagoas
}

\author{
Clóvis Isaac Silva de $\operatorname{Lima}^{1}$ (D), Fabrício Daniel dos Santos Silva ${ }^{1}$ (D), \\ Ismael Guidson Farias de Freitas $^{1}$ (D), David Duarte Cavalcante Pinto ${ }^{1}$ (D), \\ Rafaela Lisboa $\operatorname{Costa}^{1}$ (D), Heliofábio Barros Gomes ${ }^{1}$ (D), Ewerton Hallan de Lima Silva ${ }^{1}$, \\ Lindenberg Lucena da Silva ${ }^{2}$, Vicente de Paulo Rodrigues da Silva ${ }^{2}$ (D), \\ Bruce Kelly da Nóbrega Silva ${ }^{3}$ \\ ${ }^{1}$ Instituto de Ciências Atmosféricas, Universidade Federal de Alagoas, Maceió, AL, Brasil. \\ ${ }^{2}$ Unidade Acadêmica de Ciências Atmosféricas, Universidade Federal de Campina Grande, \\ Campina Grande, PB, Brasil. \\ ${ }^{3}$ Programa de Pós Graduação em Ciências Climáticas, \\ Universidade Federal do Rio Grande do Norte, Natal, RN, Brasil.
}

Recebido em: 22 de Julho de 2020 - Aceito em: 5 de Dezembro de 2020

\begin{abstract}
Resumo
O cultivo de milho em regime de sequeiro no estado de Alagoas é determinado pela variabilidade climática, especialmente sob as recorrentes secas que influenciam a recarga dos recursos hídricos em toda a região Nordeste do Brasil. Uma das formas de minimizar o risco de perdas é estabelecer uma janela climática ótima para o plantio. Nessa pesquisa utilizou-se um modelo agrometeorológico de penalização por déficit hídrico para simular a produtividade em todos os municípios de Alagoas no período de 1980 a 2015. A alta correlação entre simulações e observações, e o erro médio absoluto baixo para estações de referência validaram o modelo. Há diferentes janelas favoráveis ao plantio, mais curta no sertão durante o mês de abril, entre o terceiro decêndio de março e o terceiro decêndio de maio no agreste, e entre o primeiro decêndio de março e o segundo decêndio de junho no leste alagoano. Em média, as perdas relativas de produtividade no sertão são de $45 \%$, no agreste de $40 \%$ a $45 \%$, e em torno de $20 \%$ no leste. Estes resultados podem auxiliar o Zoneamento Agrícola de Risco Climático de Culturas do Ministério da Agricultura, Pecuária e Abastecimento, a estabelecer um calendário mais criterioso para a semeadura do milho no estado de Alagoas.
\end{abstract}

Palavras-chave: risco climático, janela de semeadura, modelagem.

\section{Alternative Method of Agroclimatic Zoning of Maize for the State of}

\begin{abstract}
Alagoas
The cultivation of corn under a rainfed regime along the state of Alagoas is deeply influenced by climate variability, especially when considering the recurring droughts affecting the entire Northeast region of Brazil. One way of minimizing the risk of losses is establishing a climate window that is optimal for the sowing time. This study resorted to an agrometeorological model of damages by water deficit to simulate the productivity in every municipality of Alagoas from 1980 to 2015. The high correlation between simulations and observations, and the low absolute mean error for reference stations validated the model. There are different windows favourable to planting, shorter in the hinterland during the month of April, between the third ten days of March and the third ten days of May in the wild, and between the first ten days of March and the second ten days of June in eastern Alagoas. On average, relative productivity losses are about $45 \%$ in "Sertão", $40-45 \%$ in "Agreste", and around 20\% in the coast. Such results can aid the Brazilian Ministry of Agriculture, Livestock, and Supply, in the elaboration of its Agricultural Climate Risk Zoning of Crops, potentially resulting in a more considerate calendar for corn sowing in Alagoas.
\end{abstract}

Keywords: climate risk, sowing window, modeling.

Autor de correspondência: Clóvis Isaac Silva de Lima, clovis.lima@icat.ufal.br. 


\section{Introdução}

O milho é uma das culturas mais antigas do mundo, sendo até hoje amplamente cultivada, e desempenha papel fundamental no sistema de produção alimentar brasileiro (Vorpagel, 2010; Martins, 2012). O elevado potencial produtivo, composição química e valor nutritivo fazem com que esse cereal seja considerado mundialmente como um dos mais importantes, e devido a sua versatilidade de uso, é utilizado tanto na indústria, como na alimentação humana e animal (Vorpagel, 2010).

Há uma grande diversidade nas condições de cultivo do milho no Brasil, e devido a isto, se observa uma grande parcela de pequenos produtores com agricultura tipicamente de subsistência, sem utilização de insumos modernos, cuja produção é voltada para consumo na propriedade e eventual excedente comercializado (Duarte et al., 2010), e uma pequena parcela de grandes produtores com alto índice de produtividade, que empregam maior área, capital e tecnologia na produção de milho, com altas produtividades equivalentes às obtidas em países de agricultura mais avançada (dos Reis et al., 2016).

No caso do Nordeste, que tem a maior parte de sua área inserida no clima semiárido, o aumento na ETP associado a alta variabilidade espaço-temporal da precipitação, pode prejudicar ainda mais as atividades agrícolas, uma vez que esta tendência continua para diferentes cenários futuros de mudanças climáticas (Marengo et al., 2009; Rossato et al., 2017; Marengo et al., 2018; Brito et al., 2018).

A maior parte do território do estado de Alagoas está inserido na porção semiárida do Nordeste, uma região caracterizada por secas recorrentes (da Rocha Júnior et al., 2020). Por sua susceptibilidade ao insucesso das safras, boa parte dos agricultores são atendidos pelo Seguro da Agricultura Familiar (SEAF) do Programa Garantia Safra (GS), que visa assegurar a renda do agricultor em situações de quebra de safra decorrentes de estiagem ou excesso hídrico. Do ponto de vista da segurança alimentar, o milho é o alimento básico para a maioria da população rural alagoana.

A produtividade das culturas depende fortemente das condições climáticas, com os experimentos de campo sendo fundamentais para aprimorar modelos que consigam representar bem a interação clima e agricultura (Greatrex, 2012). Entre os objetivos de se desenvolver modelos numéricos eficientes na representação do ciclo de vida das culturas, estão a definição de datas ótimas para semeadura, aplicação de técnicas de manejo agrícola e, principalmente, previsibilidade das safras em resposta ao clima (Jame e Cutfort, 1996; Jones et al., 2003; Murthy, 2004; Assad et al., 2007; Hsiao, 2009; Lorençoni, 2010; Corrêa, 2011; Scarpare, 2011; Hoogenboom et al., 2012; García Vila e Fereres, 2012, Monteiro et al., 2017).

Uma estratégia para minimizar as perdas de safras agrícolas em áreas de extrema vulnerabilidade climática, como Alagoas, é semear no momento certo (Sans e Guimarães, 2006; Amaral et al., 2009). Nesse sentido, o Ministério da Agricultura, Pecuária e Abastecimento do Brasil (MAPA) fornece aos agricultores uma ferramenta de Zoneamento Agrícola de Risco Climático (ZARC), também usada por formuladores de políticas e agências de gerenciamento de riscos (Brasil, 2013).

$\mathrm{O}$ zoneamento é baseado principalmente em um balanço hídrico no solo, a fim de calcular índices de necessidade de água (ISNA) na fase de máxima demanda hídrica da cultura (Brunini et al., 2001; Andrade Junior et al., 2007; Andrade Junior et al., 2009; Andrade Junior et al., 2018a; Andrade Junior et al., 2018b; Andrade Junior et al., 2018c).

Esse trabalho tem por objetivo usar um método alternativo para identificar a melhor época de semeadura do milho para o estado de Alagoas baseado no histórico do rendimento médio das safras agrícolas. Para tanto, foi utilizado um modelo agrometeorológico de penalização por deficiência hídrica (Doorembos e Kassam, 1979), uma vez que a maior parte do cultivo deste grão é de sequeiro e as secas são um fenômeno climático de alta frequência que atinge o estado (da Rocha Júnior et al., 2020).

\section{Materiais e Métodos}

\subsection{Dados observados e área de estudo}

Os dados de produtividade observada do milho para o estado de Alagoas são disponibilizados pelo Instituto Brasileiro de Geografia e Estatística (IBGE), através de seu Sistema IBGE de Recuperação Automática (SIDRA). Pelo SIDRA é possível obter dados da produtividade média da produção, em quilogramas por hectare, das lavouras temporárias de milho a partir do ano de $1974 \mathrm{com}$ dados mensais. Os dados de produtividade fornecidos pelo IBGE não informam o ciclo da cultura nem a variedade plantada, desta forma, utilizou-se esta informação como sendo o rendimento obtido para determinado município considerando um ciclo médio para o milho de 120 dias.

Os dados de entrada necessários ao modelo agrometeorológico são precipitação (PREC) e evapotranspiração potencial (ETP). Foram extraídas séries temporais diárias destas variáveis para cada município do estado a partir da análise gradeada disponibilizada por Xaviet et al. (2016), relativas ao período 1980-2015, de acordo com a Fig. 1, utilizando o método de interpolação bilinear simples (Liu et al., 2015). Esse método calcula um valor da variável em um ponto específico da grade (centroide do município), atribuindo pesos característicos a cada um dos quatro pontos da grade em relação à coordenada geográfica do ponto do centroide de interesse, com um peso maior quanto mais próximo do centroide estiver o respectivo ponto da grade (da Rocha Júnior et al., 2019). A ETP disponibilizada por 


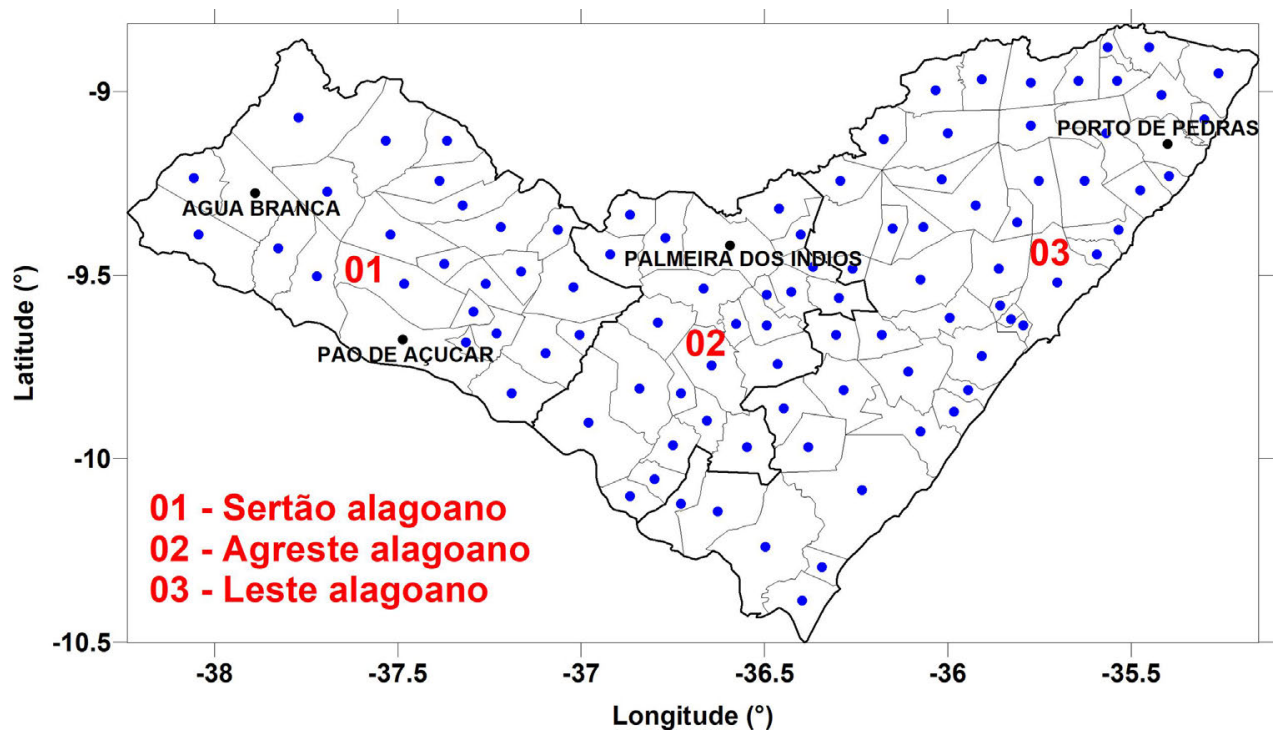

Figura 1 - Centroides dos municípios em que foram extraídas séries temporais de PREC e ETP da análise gradeada (Fonte: Xavier et al., 2016) e estações convencionais do INMET nas diferentes mesorregiões de Alagoas.

Xavier et al. (2016) foi obtida pelo método de PenmanMonteith FAO 56.

Além dos dados disponibilizados por Xavier et al. (2016), foram utilizados dados de PREC e ETP para o mesmo período de quatro estações meteorológicas do INMET, localizadas nos municípios de Água Branca e Pão de Açúcar (mesorregião do sertão alagoano), Palmeira dos Índios (mesorregião do agreste alagoano), e Porto de Pedras (mesorregião do leste alagoano). Estas estações foram usadas para validar tanto os dados provenientes da análise gradeada, como das produtividades simuladas com o modelo agrometeorológico.

\subsection{Modelo agrometeorológico de penalização por deficiência hídrica}

Foi utilizado o modelo proposto por Doorenbos e Kassan (1979), que estabelece a penalização do rendimento agrícola a partir da deficiência hídrica no decorrer das fases fenológicas da cultura. Esse modelo foi escolhido, pois a maior parte das perdas agrícolas no Nordeste brasileiro se dá por secas e/ou estiagens prolongadas, não apenas no semiárido (Alvalá et al., 2017).

Este modelo é composto por dois módulos: um que estima a produtividade potencial da safra ou produtividade máxima, e outra que estima o decréscimo do potencial de produtividade em função do déficit hídrico. O passo de tempo do modelo é decendial e as únicas entradas são a PREC e a evapotranspiração potencial da cultura $(E T P c)$. A Evapotranspiração Real da Cultura $(E T R c)$ e o coeficiente de penalização por déficit hídrico $(k y)$, são provenientes do balanço hídrico para o ciclo da cultura, mostrados na Eq. (1).

$$
Y d=\left(1-\frac{E T R c}{E T P c}\right) \times k y \times 100
$$

O ajuste do modelo foi realizado para o período 20002010, para comparar a produtividade simulada nos quatro municípios com estações do INMET e a observada, e implementar a metodologia proposta por Monteiro et al. (2013; 2017). Nesta metodologia, busca-se encontrar um valor alternativo para o $k y, \mathrm{o} k y^{*}$, a fim de representar com maior acurácia os diferentes níveis de sensibilidade ao déficit hídrico de acordo com valores históricos de produtividades reais observadas. Aliado ao $k y^{*}$, um fator de correção relativo ao rendimento tecnológico máximo que a cultura poderia atingir $(\Delta p)$ é introduzido a fim de minimizar as diferenças entre os valores simulados e observados, ambos a fim de minimizar o erro médio absoluto (EMA) entre simulações e observações aproximando-se o máximo possível as retas de tendência linear simuladas e observadas, resultando na Eq. (2) para a produtividade relativa. Além da avaliação do EMA, o coeficiente de correlação de Pearson foi usado para avaliar o grau de concordância entre as simulações e as séries históricas.

$$
Y d=\frac{P R}{P M T}=\left[1-\left(\frac{E T R c}{E T P c}\right)\right] \times k y \times k y^{*} \times 100
$$

em que $Y d$ é o rendimento potencial final ( $\%$ ou em $\left.\mathrm{kg} \mathrm{ha}^{-1}\right)$, $P M T$ é a Produtividade Máxima Tecnológica estimada a partir de um ajuste estatístico baseado na série de dados das Produtividades Reais Observadas (PR). A ETPc e a ETRc são obtidas a partir do balanço hídrico da cultura usando o método de Thornthwaite e Mather (1955), e pela multi- 
plicação entre as evapotranspirações potenciais e reais com o coeficiente da cultura (Doorenbos e Kassan, 1979)

\section{Resultados e Discussão}

\subsection{Calibração do modelo agrometeorológico}

A calibração do modelo agrometeorológico foi realizada para quatro municípios (Água Branca e Pão de Açúcar no Sertão alagoano, Palmeira dos Índios no Agreste alagoano e Porto de Pedras no Leste alagoano) com auxílio das estações meteorológicas do INMET. Nesses municípios, as produtividades médias anuais observadas no período entre 2000 e 2010, com os dados do IBGE, foram de $368 \mathrm{~kg} / \mathrm{ha}$ em Água Branca; $448 \mathrm{~kg} / \mathrm{ha}$ em Pão de Açúcar; $522 \mathrm{~kg} / \mathrm{ha}$ em Palmeira dos Índios, e $629 \mathrm{~kg} / \mathrm{ha}$ em Porto de Pedras. Nota-se que a produtividade média é inferior a $1000 \mathrm{~kg} / \mathrm{ha}$, o que caracteriza o milho como um cultivo de baixa produtividade no estado de Alagoas (CONAB, 2019).

Além disso, observa-se um aumento da produtividade do milho da mesorregião do Sertão ao Leste alagoano. Essa variabilidade reflete as condições climáticas do estado, com um gradiente de precipitação zonal do litoral (maior acumulação anual) ao sertão (menor acumulação anual), como mostra a Fig. 2, além da maior demanda atmosférica por água de sistemas solo-plantaatmosfera no sertão, seguido por Agreste e Leste alagoanos (Lyra et al., 2014; Lyra et al., 2015; Silva et al., 2018). No caso do milho em Alagoas, predomina a agricultura familiar de subsistência, em regime de sequeiro, sujeita a alta variabilidade espaço-temporal das chuvas e a recorrência de extremos climáticos extremos (da Rocha Júnior, 2019; da Rocha Júnior, 2020; Costa et al., 2020).

Nesta perspectiva, foram calculados o coeficiente de rendimento máximo tecnológico $(\Delta p)$ e o fator de déficit hídrico ajustado $\left(k y^{*}\right)$ para cada município, durante o pro- cesso de calibração do modelo de produtividade. Estes parâmetros permitiram simular o rendimento com maior precisão em cada município.

Na Fig. 3 observa-se que Palmeira dos Índios, Porto de Pedras e Pão de Açúcar apresentaram as maiores correlações entre os dados observados e simulados pelo modelo, 0,78, 0,69 e 0,81 respectivamente, e EMA de $38 \mathrm{~kg} / \mathrm{ha}$, $84 \mathrm{~kg} / \mathrm{ha}$ e $71 \mathrm{~kg} / \mathrm{ha}$. No município de Água Branca o EMA foi de $136 \mathrm{~kg} / \mathrm{ha}$ e a correlação igual a 0,59 .

Tais resultados demonstram que o modelo tende a se ajustar à variabilidade anual dos dados observados, com média do período próxima a média observada e EMA dentro do esperado, sem se distanciar de forma desfavorável em relação aos valores observados em uma simulação. Percebe-se ainda na Fig. 3, a tendência positiva da produtividade na reta de mínimos quadrados dos dados do IBGE, e o potencial de rendimento crescente atingível representado pela linha da tendência da produtividade potencial tecnológica.

\subsection{Zoneamento agroclimático}

Os resultados exibidos no tópico anterior demonstram a confiança em se utilizar o modelo, e os resultados obtidos levaram em conta as datas de plantio preconizadas pelo Governo Federal em sua Portaria de $\mathrm{N}^{\circ} 167$ de 2 de outubro de 2013, publicado pela Secretaria de Política Agrícola do Ministério da Agricultura Pecuária e Abastecimento (MAPA), que estabeleceu o zoneamento agrícola do risco climático para a cultura do milho no estado de Alagoas, e o respectivo calendário agrícola da semeadura da cultura, onde se recomenda o período de plantio entre 20 de março e 21 de junho (CONAB, 2019), com três decêndios preferenciais de plantio: 20/04, 20/05 e 20/06.

No entanto, foram examinados se estes seriam realmente os melhores decêndios para a semeadura, ou seja, as que climaticamente, levam a uma maior produtividade.

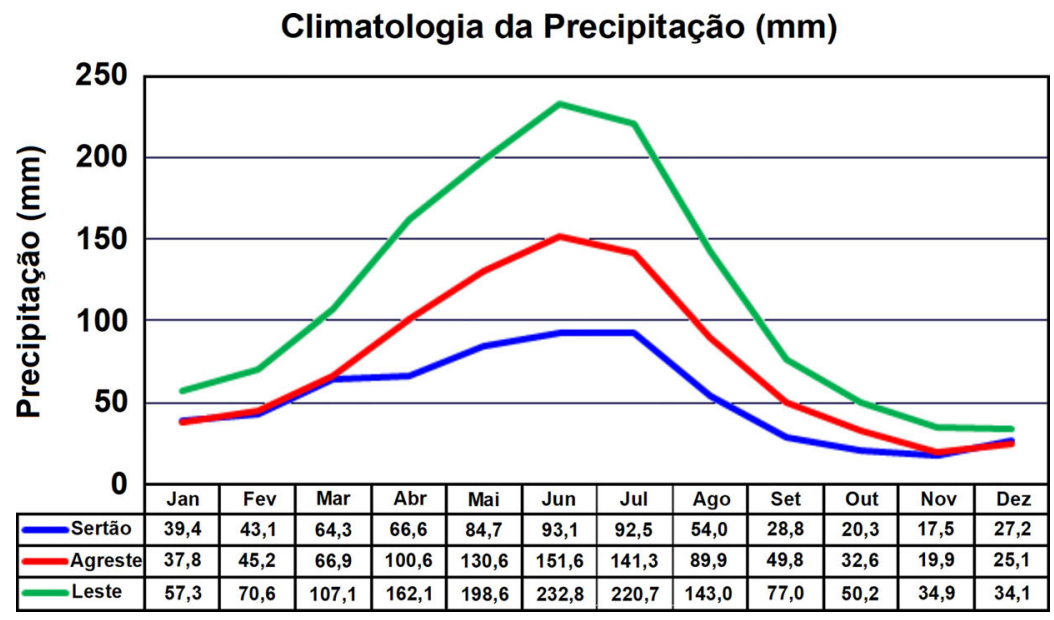

Figura 2 - Climatologia da precipitação nas mesorregiões alagoanas: Sertão (linha azul), Agreste (linha vermelha) e Leste (linha verde). 

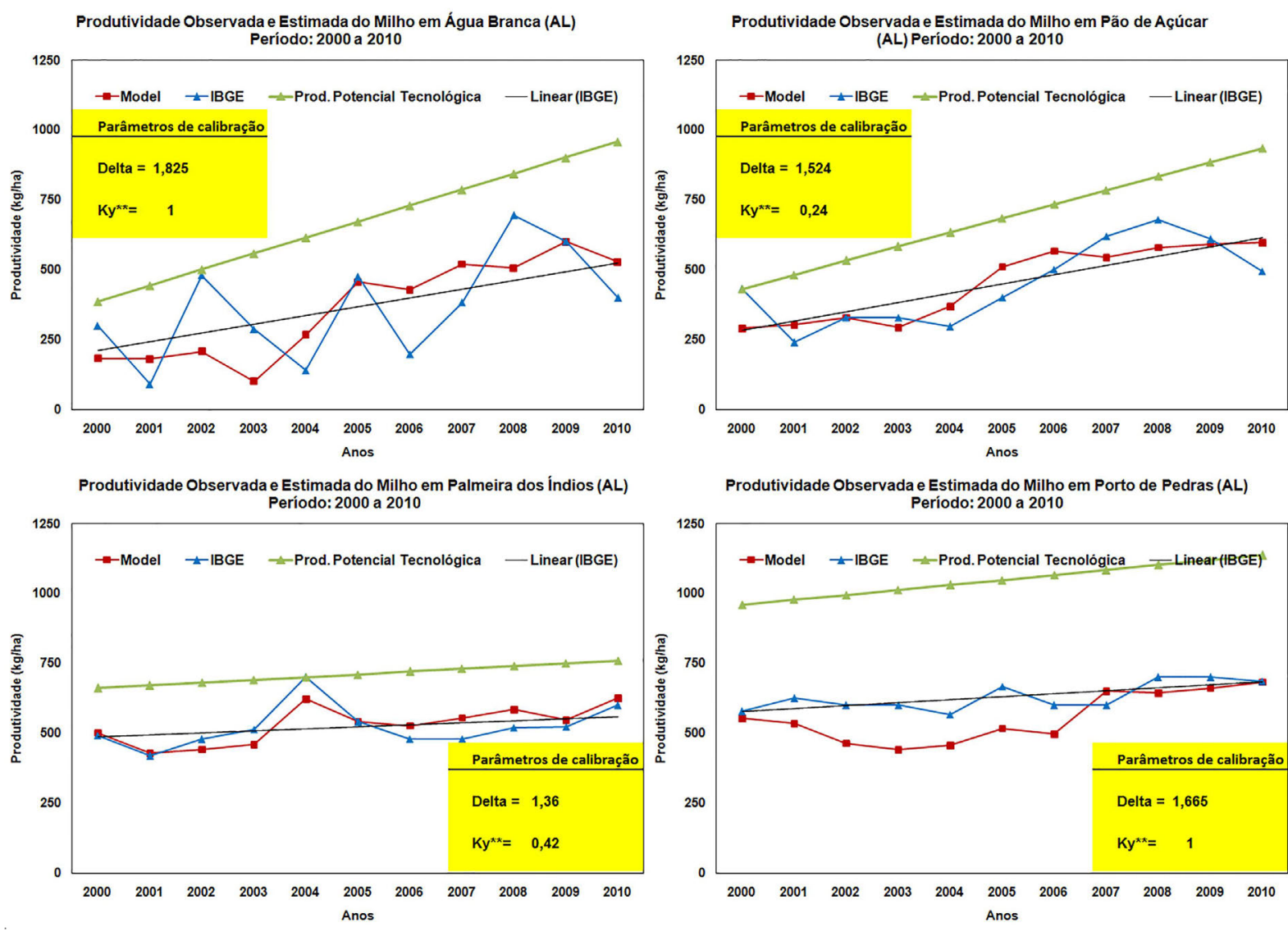

Figura 3 - Produtividades observadas e simuladas do milho nas cidades Água Branca, Pão de Açúcar, Palmeira dos Índios e Porto de Pedras.

Para tanto, a partir da base de dados disponibilizada por Xavier et al. (2016), para cada município foi obtida as séries temporais decendiais de precipitação e ETP relativas ao período 1980-2015, e para cada decêndio do ano foram simulados os rendimentos relativos (\%), permitindo obter médias anuais da produtividade em cada município e desta forma estabelecer o período em decêndios mais apto a semeadura.

Esta metodologia reversa de estimativa de produtividade a partir de modelagem agrometeorológica aplicada a um período de referência foi utilizada por Paixão et al. (2014), também para o milho em condições de sequeiro, para a determinação de uma melhor janela de semeadura a fim de reduzir os impactos naturais da variabilidade climática anual e interanual na produtividade. $\mathrm{O}$ modelo utilizado foi o CSM-Ceres-Maize e o local analisado foi o estado Minas Gerais. Os resultados indicaram que este tipo de modelagem traz vantagens em relação às metodologias adotadas pelo MAPA, permitindo integrar fatores não consideradas no zoneamento tradicional, além da possibilidade de determinar o rendimento médio esperado e a sua amplitude.

Monteiro et al. (2013) aplicaram o mesmo modelo agrometeorológico dessa pesquisa para avaliar a perda de produtividade por deficiência hídrica em diferentes localidades brasileiras, e concluíram que, quando o modelo indica perda relativa superior a $50 \%$, em mais de $90 \%$ dos casos as perdas em campo são comprovadas por laudos técnico do IBGE, preparados por técnicos agrícolas in loco. De acordo com este resultado, utilizou-se nos resultados das Figuras 3 a 14 uma escala inversa, mostrando a variação de produtividade percentual atingível de 0 a $100 \%$, ou seja, tons em vermelho indicam menores valores de produtividade relativa e valores acima de $60 \%$, em tons de verde e azul, os que indicam maior probabilidade de que se alcance uma boa produtividade. Foi usado o valor de $60 \%$ em vez de 50\% como em Monteiro et al. (2013), a fim de garantir uma maior probabilidade associada ao sucesso das safras, uma vez que o estudo de Monteiro et al. (2013) foi aplicado a diferentes localidades em todo o Brasil, e este estudo se aplica apenas a um estado.

Os resultados são provenientes das médias de produtividade simuladas para cada decêndio no período 19802015, ou seja, 36 valores para composição da média por decêndio. A primeira percepção desses resultados referese aos períodos desfavoráveis ao cultivo, em todos os decêndios de janeiro (Fig. 4) e fevereiro (Fig. 5), e a partir de julho até dezembro. 

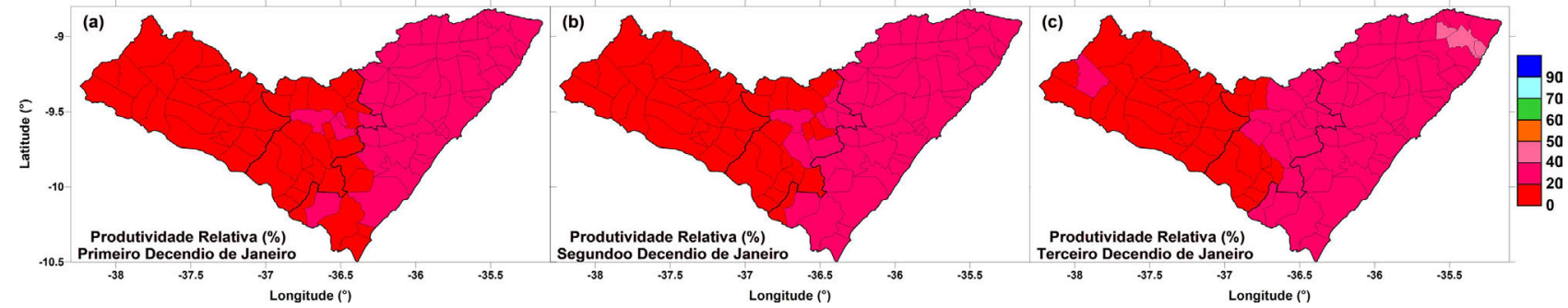

Figura 4 - Produtividade relativa percentual média do milho para o (a) primeiro, (b) segundo e (c) terceiro decêndios de janeiro.
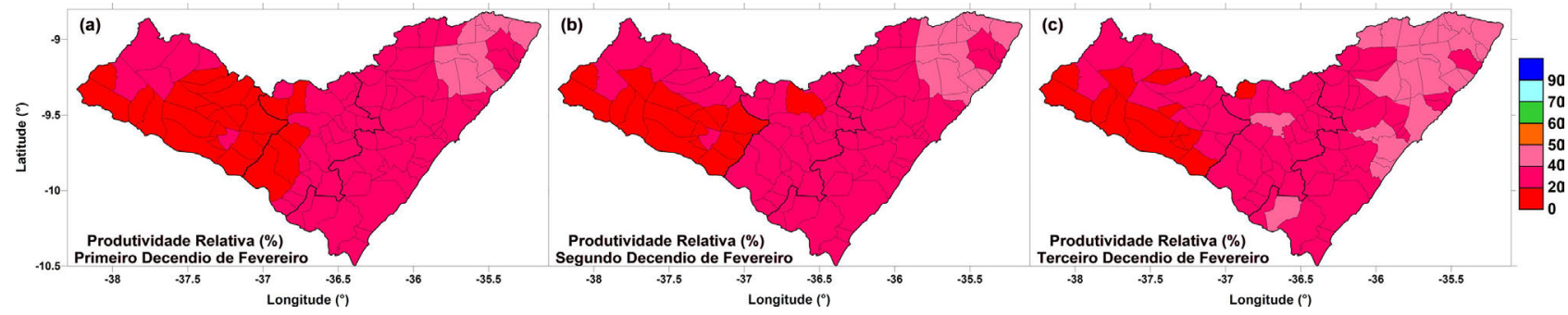

Figura 5 - O mesmo da Figura 3, mas para fevereiro.

A partir do primeiro decêndio de março (Fig. 6a), condições favoráveis para o cultivo já são visíveis no Leste alagoano, respaldado por simulações que já indicam a possibilidade de rendimentos superiores a $60 \%$, fortalecendo-se no segundo e terceiro decêndios (Figuras $6 \mathrm{~b}$ e $6 \mathrm{c}$ ), avançando gradativamente para o Agreste e Sertão alagoano a partir de abril (Fig. 7) e maio (Fig. 8), com destaque entre o primeiro decêndio de abril (Fig. 7a) ao primeiro decêndio de maio (Fig. 8a), no entanto, com rendimentos variáveis entre as regiões, ou seja, maiores no Leste do que no Agreste e no Sertão, gradativamente. Os dois primeiros decêndios de junho ainda são favoráveis ao cultivo na faixa Leste e alguns municípios do Agreste (Figs. 9a e 9b), perdendo intensidade e sendo favorável apenas em alguns municípios da mesorregião Leste no terceiro decêndio de junho (Fig. 9c). Percebe-se visualmente que a janela de semeadura mais favorável à obtenção de maiores produtividades é mais larga na mesorregião Leste, e menores no Agreste e Sertão, respectivamente. De julho a dezembro (Figs. 10 a 15), retoma-se a condição de meses totalmente desfavoráveis ao cultivo.
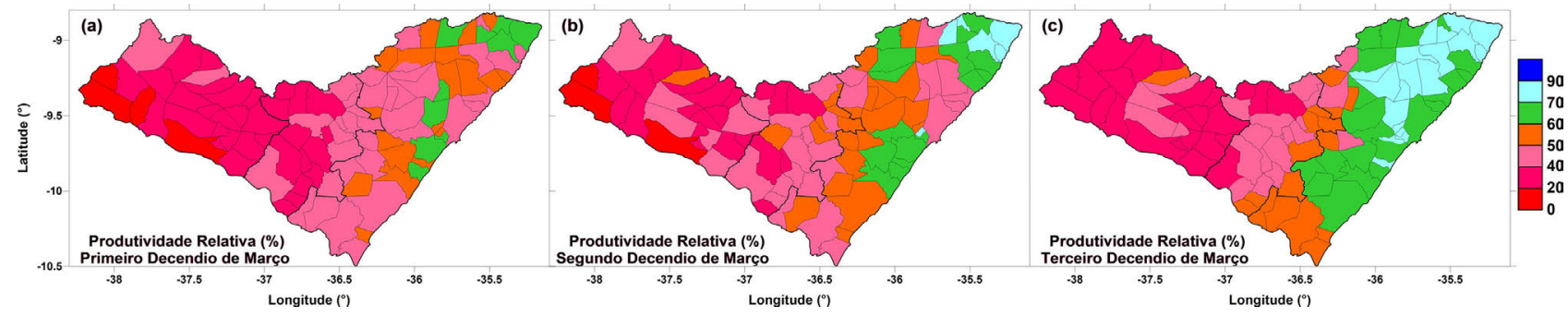

Figura 6 - O mesmo da Figura 3, mas para março.
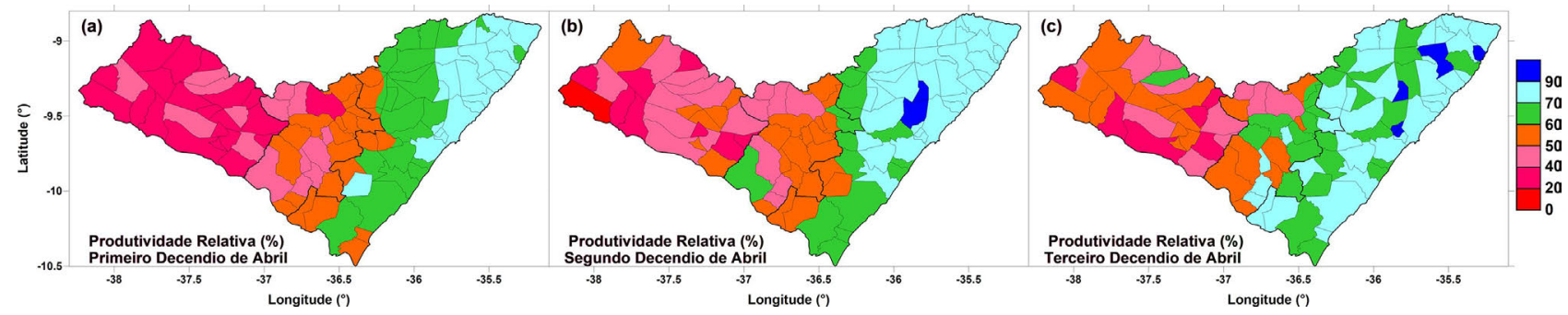

Figura 7 - O mesmo da Figura 3, mas para abril. 

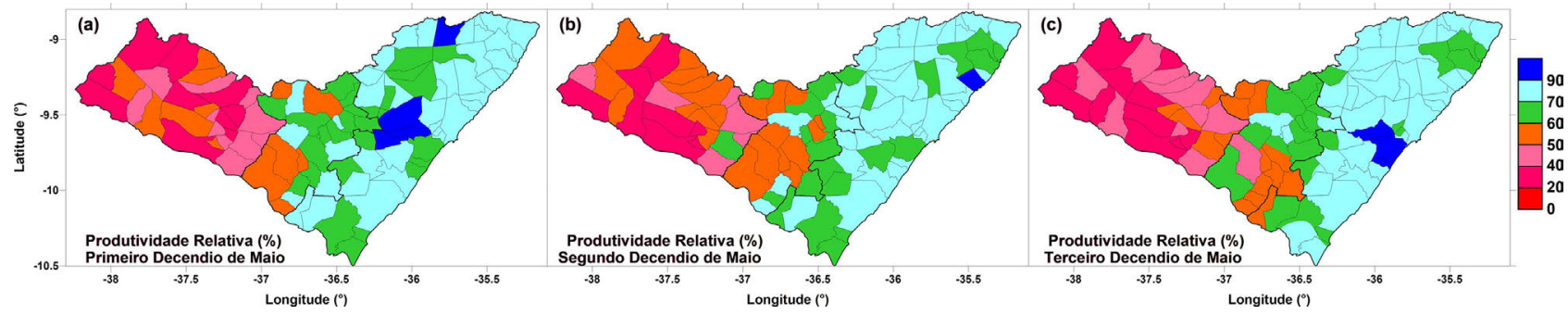

Figura 8 - O mesmo da Figura 3, mas para maio.
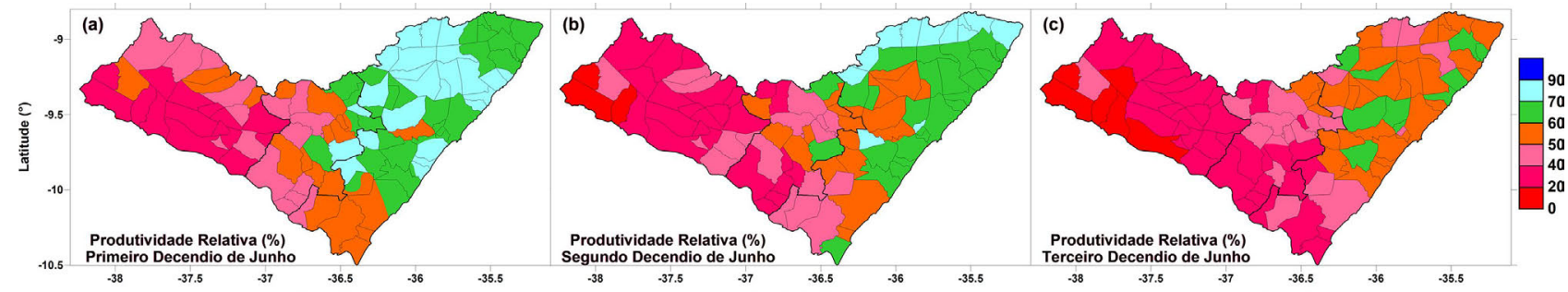

Longitude ( $\left(^{\circ}\right)$

Figura 9 - $\mathrm{O}$ mesmo da Figura 3, mas para junho.
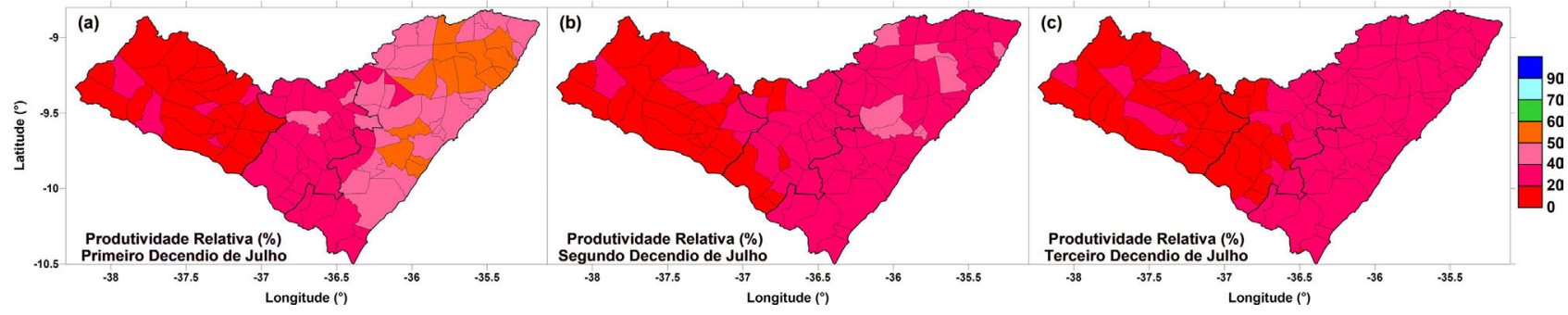

Figura 10 - O mesmo da Figura 3, mas para julho.
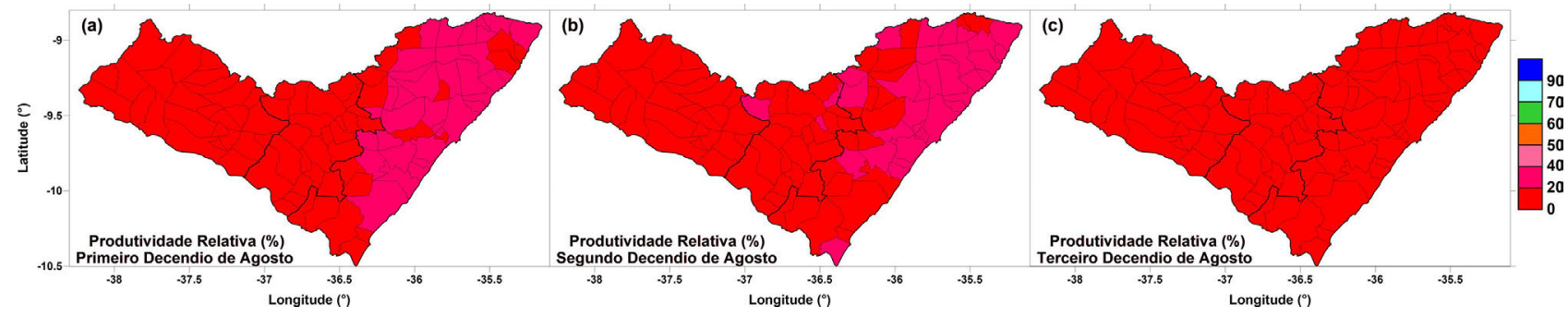

Figura 11 - O mesmo da Figura 3, mas para agosto.
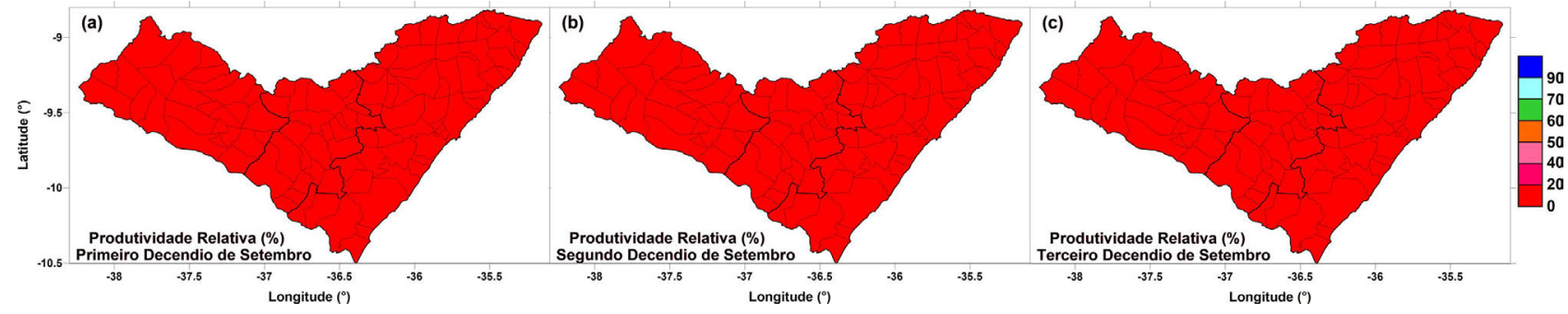

Figura 12 - O mesmo da Figura 3, mas para setembro. 


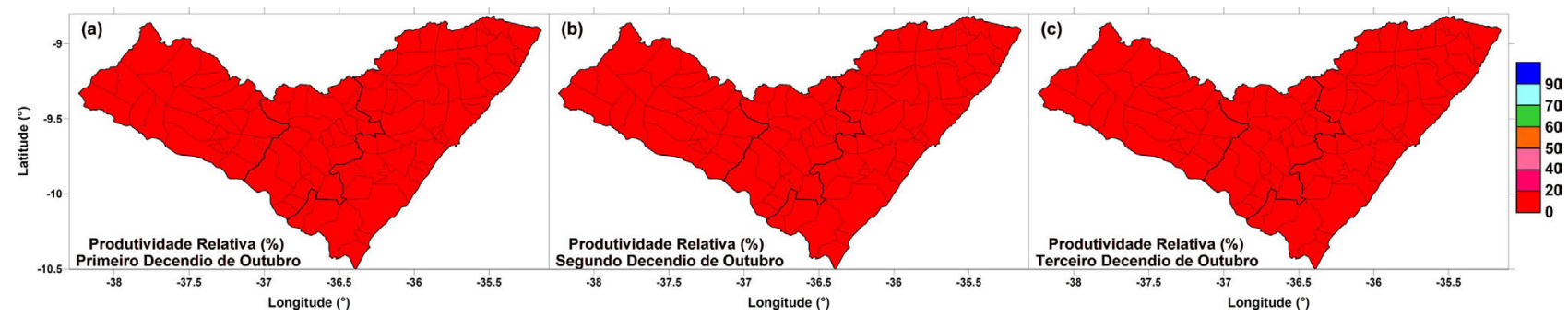

Figura 13 - O mesmo da Figura 3, mas para outubro.
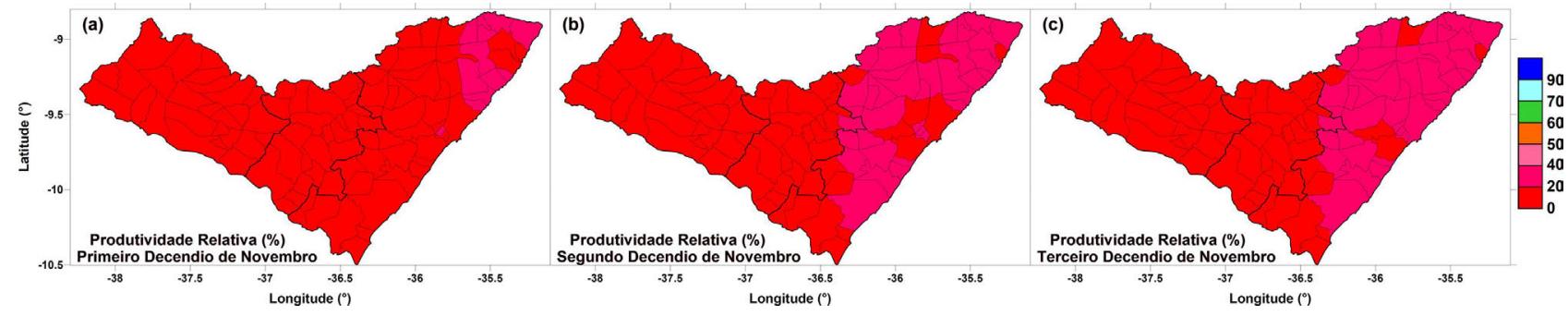

Figura 14 - O mesmo da Figura 3, mas para novembro.
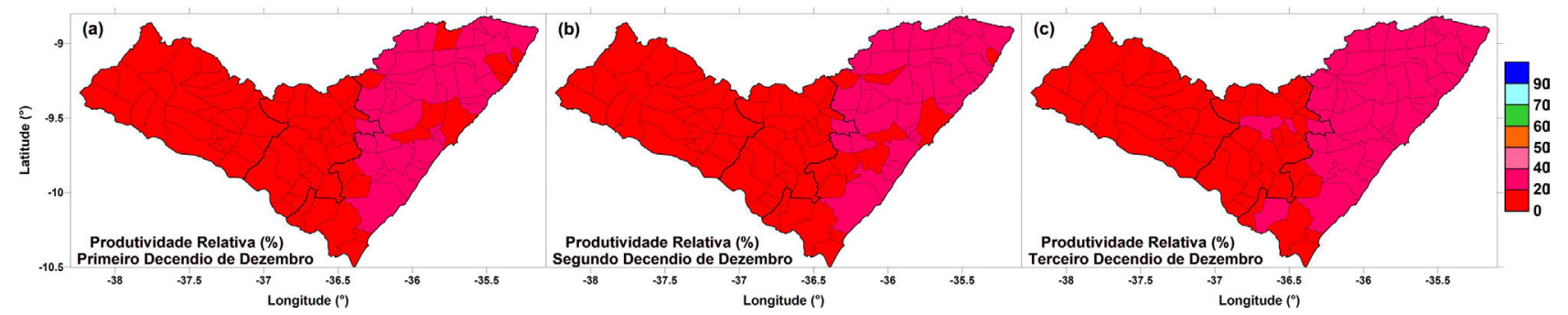

Figura 15 - O mesmo da Figura 3, mas para dezembro.

Como se pôde observar, o mapeamento traz boa noção das melhores janelas de favorabilidade à semeadura do milho nas diferentes mesorregiões. Mas ainda se torna tarefa difícil estabelecer a época de plantio para uma dada mesorregião, dada a variabilidade entre municípios como ocorre nas mesorregiões do Agreste e Sertão alagoano.

Para facilitar este processo de determinação de um calendário ótimo de semeadura em cada mesorregião, foi considerado como favoráveis os decêndios do ano que conseguem superar em, ao menos, $50 \%$ a produtividade relativa (linha azul da Fig. 16). Para tanto, em cada mesorregião, os resultados foram compilados a partir da média dos dados dos municípios que as compõem. No caso do Sertão, o resultado da produtividade potencial do milho foi obtido dos valores médios de 28 municípios, para o agreste de 21 municípios e para o leste alagoano de 53 municípios, totalizando os 102 municípios que compõe o estado.

Com esses resultados, a Fig. 16 possibilita identificar que a produtividade do milho, limita-se ao período de março ao primeiro decêndio de junho, em média, quando as condições climáticas neste período são favoráveis devi- do a presença da estação chuvosa, e temperaturas ideais para o desenvolvimento do cultivo. Por outro lado, entre o segundo decêndio de junho a fevereiro é encontrado baixa disponibilidade hídrica, somada a elevadas temperaturas, que dificulta o desenvolvimento do milho nas atividades de sequeiro. (Fig. 16a).

O Agreste alagoano apresenta alta vulnerabilidade às perdas, com produtividades relativas em torno de $55 \%$ a $60 \%$, porém com maior janela apta à semeadura, entre o terceiro decêndio de março e o terceiro decêndio de maio (Fig. 16b). Com isso pode-se concluir que essa zona de transição tem um potencial considerável sendo possível desenvolver técnicas para diminuir a carência de água realizando inovações nos processos de gerenciamento hídrico que podem colaborar para a garantia da safra plantada entre esses decêndios do ano.

O Leste alagoano possui a maior janela de plantio, entre o primeiro decêndio de março e o segundo decêndio de junho, e em média com as maiores produtividades relativas, podendo atingir o patamar de 80\% (Fig. 16c). Além disso, essa faixa tem o benefício geográfico de sua localização que por sua vez possui maiores índices pluviomé- 
(a) Produtividade potencial média do milho para diferentes épocas de semeadura - Sertão Alagoano

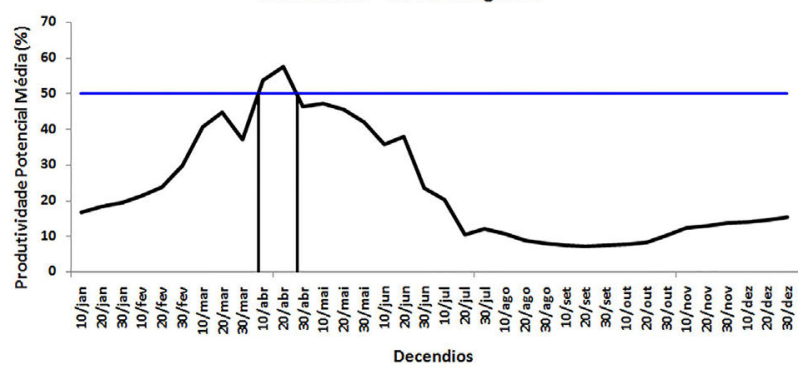

(b) Produtividade potencial média do milho para diferentes épocas de semeadura - Agreste Alagoano

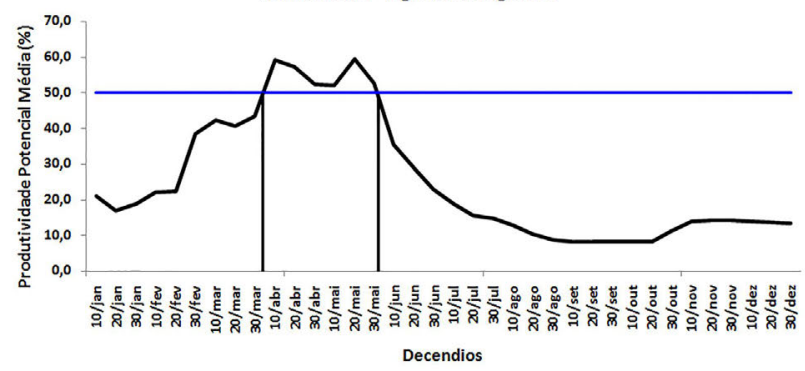

(c) Produtividade potencial média do milho para diferentes épocas de semeadura - Leste Alagoano

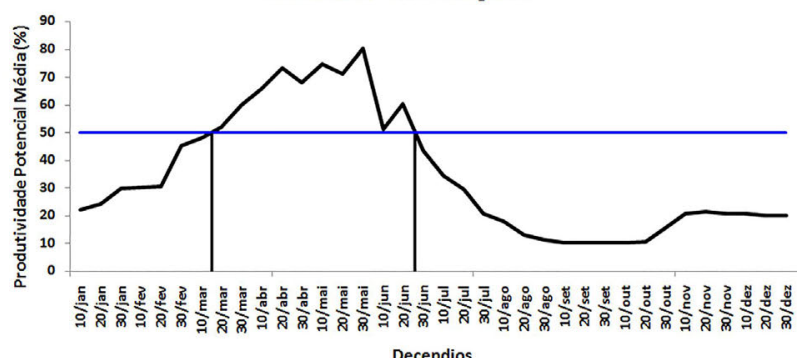

Decendios

Figura 16 - Produtividade potencial média do milho para as diferentes mesorregiões do estado de Alagoas: (a) sertão alagoano, (b) agreste alagoano e (c) leste alagoano.

tricos, podendo ser mais explorado o seu potencial agrícola utilizando-se processos de mecanização que venham colaborar com a melhora dos índices socioeconômico dessa mesorregião.

Os resultados mostrados na Fig. 16 permitem inferir, ainda, que a diminuição progressiva das precipitações a partir de julho/agosto e o aumento das temperaturas a partir de agosto/setembro, e consequentemente da ETP, são os fatores limitantes ao cultivo de milho entre julho a fevereiro.

\section{Conclusões}

O modelo agrometeorológico de penalização por deficiência hídrica empregado para estimativa da produtividade do milho no estado de Alagoas apresentou correlações satisfatórias e erro médio absoluto considerado baixo na relação simulação-observação.
Este mesmo modelo, ao ser usado para dados climáticos de todos os municípios, permitiu identificar por meio de um histórico de 36 anos de simulações, os melhores decêndios para o cultivo do milho no estado de Alagoas. Segundo o calendário mais recente de plantio e colheita no Brasil, disponibilizado pela CONAB (2019), o milho cultivado em Alagoas faz parte do calendário de segunda safra, e deve ser semeado no outono-inverno entre abril e julho, para ser colhido entre agosto e dezembro. Os resultados desta pesquisa contrastam com esta recomendação, pois demonstrou-se que as três mesorregiões do estado possuem diferentes janelas favoráveis a semeadura, mais curta no Sertão, onde o produtor poderá atingir o máximo de produtividade plantando durante o mês de abril; intermediária no Agreste, onde o produtor poderá atingir o máximo de produtividade plantando entre o terceiro decêndio de março e o terceiro decêndio de maio; e mais ampla no leste alagoano, onde o produtor poderá atingir o máximo de produtividade plantando entre o primeiro decêndio de março e o segundo decêndio de junho.

Além de uma variabilidade natural entre os períodos mais aptos à semeadura nas diferentes mesorregiões, os níveis de produtividade potencial médios diferem entre as mesmas, com rendimento médio percentual de aproximadamente $55 \%$ no Sertão (percentual médio de perda de $45 \%$ ), $55 \%$ a $60 \%$ no agreste (percentual médio de perda de 40 a $45 \%$ ), e próximo a $80 \%$ na faixa Leste (percentual médio de perda de 20\%).

Espera-se que tais resultados sirvam de informação para a readequação do calendário de plantio e colheitas para as diferentes mesorregiões de Alagoas, e que esta pesquisa possa ser ampliada para mais estados da região Nordeste do Brasil.

\section{Agradecimentos}

Os autores agradecem a Coordenação de Aperfeiçoamento de Pessoal de Nível Superior (CAPES) pelo apoio financeiro concedido durante a concepção deste estudo. O primeiro autor agradece especialmente a Fundação de Amparo à Pesquisa do Estado de Alagoas (FAPEAL), pela concessão da bolsa de estudos, processo $\mathrm{n}^{\circ}$ 60030.0011094/2018.

\section{Referências}

AMARAL, T.A.; ANDRADE C.L.T.; OLIVEIRA, A.C.; SILVA, D.F.; SANTANA, C.B.; MOURA, B.F.; CASTRO, L.A. Metodologia para o Estabelecimento do Período de Semeadura de Milho. Sete Lagoas: Embrapa Milho e Sorgo, 13 p., 2009.

ALVALA, R.C.S.; CUNHA, A.P.M.A.; BRITO, S.S.B.; SELUCHI, M.E.; MARENGO, J.A.; MORAES, O.L.L.; CARVALHO, M.A. Drought monitoring in the Brazilian 
Semiarid region. Anais da Academia Brasileira de Ciências, v. 91, n. 1 (sup-1), p. 1-15, 2017.

AMARAL, T.A.; ANDRADE C.L.T.; OLIVEIRA, A.C.; SILVA, D.F.; SANTANA, C.B.; MOURA, B.F.; CASTRO, L.A. Metodologia para o Estabelecimento do Período de Semeadura de Milho. Sete Lagoas: Embrapa Milho e Sorgo, 13 p., 2009.

ANDRADE JÚNIOR, A.S.; BARROS, A.H.C.; SILVA, C.O.; FREIRE FILHO, F.R. Zoneamento de risco climático para a cultura do feijão-caupi no estado do Ceará. Revista Ciência Agronômica, v. 38, n. 1, p. 109-117, 2007.

ANDRADE JÚNIOR, A.S.; BASTOS, E.A.; CARDOSO, M.J.; SILVA, C.O. Zoneamento de Risco Climático para as Culturas de Milho e Feijão-Caupi Consorciadas no Estado do Piauí. Teresina: Embrapa Meio-Norte, 34 p., 2009.

ANDRADE JÚNIOR, A.S.; BASTOS, E.A.; MONTEIRO, M.M.S.; SILVA JUNIOR, J.S. da Evapotranspiração e coeficiente de cultura do feijão-caupi sob sistema de cultivo convencional e plantio direto. Agrometeoros, v. 26, n. 1, p. 191-199, 2018a.

ANDRADE JÚNIOR, A.S.; BASTOS, E.A.; SILVA, M.V.P.; SILVA JÚNIOR, J.S.; MONTEIRO, J.E.B.A. Índice de satisfação da necessidade de água do feijão-caupi sob sistema de cultivo convencional e plantio direto. Agrometeoros, v. 26, n. 1, p. 201-211, 2018 b.

ANDRADE JÚNIOR, A.S.; BASTOS, E.A.; MONTEIRO, J.E.B.A. Zoneamento Agrícola de Risco Climático para o Feijão-Caupi em Cultivo Convencional e Plantio Direto no Estado do Piauí. Teresina: Embrapa Meio-Norte, 26 p., $2018 \mathrm{c}$.

ASSAD, E.D.; MARIN, F.R.; EVANGELISTA, S.R.; PILAU, F.G.; FARIAS, J.R.B.; PINTO, H.S.; ZULLO JÚNIOR, J. Sistema de previsão da safra de soja para o Brasil. Pesquisa Agropecuária Brasileira, v. 42, n. 5, p. 615-625, 2007.

BRASIL. Ministério da Agricultura, Pecuária e Abastecimentos. Zoneamento Agrícola de Risco Climático. Disponível em http://https://www.gov.br/agricultura/pt-br/assuntos/politica-agricola/outras-publicacoes/zoneamento-agricola.pdf/ view, acesso em 01 jun. 2020.

BRITO, S.S.B.; CUNHA, A.P.M.A.; CUNNINGHAM, C.C.; ALVALÁ, R.C.; MARENGO, J.A.; CARVALHO, M.A. Frequency, duration and severity of drought in the Semiarid Northeast Brazil region. International Journal of Climatology, v. 38, n. 2, p. 517-529, 2018.

BRUNINI, O.; ZULLO JR, J.; PINTO, H.D.; ASSAD, E.; SAWAZAKI, E.; DUARTE, A.P.; PATTERNIANI, E.Z. Riscos climáticos para a cultura de milho no estado de São Paulo. Revista Brasileira de Agrometeorologia, v. 9, n. 3, p. 519-526, 2001.

CONAB. Revista Indicadores da Agropecuária. Disponível em https://www.conab.gov.br/info-agro/precos/revistaindicadores-da-agropecuaria, acessado em 30 de ago. 2019.

CORREAA, S.T.R.; DOURADO-NETO, D.; LORENÇONI, R.; SCARPARE, F.V.; VIVIAN, R.; RUIZ, E.T. Aplicações e limitações da modelagem em agricultura - Revisão. Revista de Agricultura, v. 86, n. 1, p. 1-13, 2011.

COSTA, R.L.; BAPTISTA, G.M.M.; GOMES, H.B.; SILVA, F.D.S.; ROCHA JUNIOR, R.L.; SALVADOR, M.A.; HERDIES, D.L. Analysis of climate extremes indices over northeast Brazil from 1961 to 2014. Weather and Climate Extremes, v. 28, p. 100254, 2020.

DA ROCHA JÚNIOR, R.L.; DOS SANTOS SILVA, F.D; COSTA, R.L; GOMES, H.B; HERDIES, D.L.; SILVA, V.P.R; XAVIER, A.C. Analysis of the Space-Temporal Trends of Wet Conditions in the Different Rainy Seasons of Brazilian Northeast by Quantile Regression and Bootstrap Test. Geosciences, v. 9, n. 11, p. 457, 2019.

DA ROCHA JÚNIOR, R.L.; DOS SANTOS SILVA, F.D.; COSTA, R.L.; GOMES, H.B.; PINTO, D.D.C.; HERDIES, D.L. Bivariate Assessment of Drought Return Periods and Frequency in Brazilian Northeast Using Joint Distribution by Copula Method. Geosciences, v. 10, n. 4, p. 135, 2020.

DOORENBOS, J.; KASSAM, A.H. Yield Response Towater. Irrigation and Drainage, Paper 33. Roma: FAO, 179 p., 1979.

DOS REIS, J.G.M.; VENDRAMETTO, O.; NAAS, I.A.; COSTABILE, L.T.; MACHADO, S.T. Avaliação das Estratégias de Comercialização do Milho em MS Aplicando o Analytic Hierarchy Process (AHP). Revista de Economia e Sociologia Rural, v. 54, n. 1, p. 131-146, 2016.

DUARTE, J.O.; CRUZ, J.C.; GARCIA, J.C.; MATTOSO, M.J. Economia da produção. In: CRUZ, J.C. (ed). Cultivo do milho. 6. ed. Sete Lagoas: Embrapa Milho e Sorgo, 2010.

GARCÍA-VILA, R.; FERERES, E. Combining the simulation crop model AquaCrop with an economic model for the optimization of irrigation management at farm level. European Journal of Agronomy, v. 36, n. 1, p. 21-31, 2012.

GREATREX, H. The Application of Seasonal Rainfall Forecasts and Satellite Rainfall Estimates to Seasonal Crop Yield Forecasting for Africa. Doctoral Thesis, University of Reading, 2012.

HOOGENBOOM, G., JONES, P.W. WILKENS, C.H.; PORTER, K.J.; BOOTE, L.A.; HUNT, U.; SINGH, J.L.; LIZASO, J.W.; WHITE, O.; URYASEV, F.S.; ROYCE, R.; OGOSHI, A.J. GIJSMAN, G.Y.; TSUJI, KOO, J. Decision Support System for Agrotechnology Transfer (DSSAT) Version 4.5. University of Hawaii, Honolulu, 2012.

HSIAO, T.S.; HENG, L.; STEDUTO, P.; ROJAS-LARA, B.; RAES, D.; FERERES, E. The FAO Crop Model to Simulate Yield Response to Water: III. Parameterization and Testing for Maize. Agronomy Journal, v. 101, n. 3, p. 448459,2009.

JAME, Y.W.; CUTFORTH, H.W. Crop Growth models for decision support systems. Canadian Journal of Plant Science, v. 76, n. 1, p. 9-19, 1996.

JONES, J.W.; HOOGENBOOM, G.; PORTER, C.H.; BOOTE, K.J.; BATCHELOR, W.D.; HUNT, L.A.; WILKENS, P.W.; SINGH, U.; GIJSMAN, A.J.; RITCHIE, J.T. DSSAT cropping system model. European Journal of Agronomy, v. 18, n. 3-4, p. 235-265, 2003.

LIU, B.; LIU, F.; WANG, C.; MEI, S. Unit commitment considering flexibility and uncertainty of wind power generation. Power System Technology, v. 39, n. 3, p. 730-736, 2015.

LORENÇONI, R.; DOURADO-NETO, D.; HEINEMANN, A.B. Calibração e avaliação do modelo ORYZA-APSIM para o arroz de terras altas no Brasil. Revista Ciência Agronômica, v. 41, n. 4, p. 605-613, 2010. 
LYRA, G.B.; OLIVEIRA JÚNIOR, J.F.; ZERI, M. Cluster analysis applied to the spatial and temporal variability of monthly rainfall in Alagoas state, Northeast of Brazil. International Journal of Climatology, v. 34, n. 13, p. 3546-3558, 2014.

LYRA, G.B.; ZANETTI, S.S.; SANTOS, A.A.R.; SOUZA, J.L.; LYRA, G.B.; OLIVEIRA JÚNIOR, J.F.; LEMES, M.A.M. Estimation of monthly global solar irradiation using the Hargreaves-Samani model and an artificial neural network for the state of Alagoas in northeastern Brazil. Theoretical and Applied Climatology, v. 125, n. 3-4, p. 743-756, 2015.

MARTINS, M.A. Estimativa de Produtividade das Culturas do Milho e do Sorgo a Partir de Modelos Agrometeorológicos em Algumas Localidades da Região Nordeste do Brasil. Dissertação de Mestrado em Meteorologia, Instituto Nacional de Pesquisas Espaciais, São José dos Campos, 64 p., 2012.

MARENGO, J.A.; JONES, R.; ALVES, L.M.; VALVERDE, M.C. Future change of temperature and precipitation extremes in South America as derived from the PRECIS regional climate modeling system. International Journal of Climatology, v. 29, n. 15, p. 2241-2255, 2009.

MARENGO, J.A.; ALVES, L.M.; ALVALA, R.C.S.; CUNHA, A.P.; BRITO, S.; MORAES, O.L.L. Climatic characteristics of the 2010-2016 drought in the semiarid Northeast Brazil region. Anais da Academia Brasileira de Ciencias, v. 90, n. 2 , p. $1973-1985,2018$.

MONTEIRO, J.E.B.A.; AZEVEDO, L.C.; ASSAD, E.D.; SENTELHAS, P.C. Rice yield estimation based on weather conditions and on technological level of production systems in Brazil. Pesquisa Agropecuária Brasileira, v. 48, n. 2, p. 123-131, 2013.

MONTEIRO, J.E.B.A.; ASSAD, E.D.; SENTELHAS, P.C.; AZEVEDO, L.C. Modeling of corn yield in Brazil as a function of meteorological conditions and technological level. Pesquisa Agropecuaria Brasileira, v. 52, n. 3, p. 137-148, 2017.

MURTHY, V.R.K. Crop growth modeling and its applications in agricultural meteorology. In: SIVAKUMAR, M.V.K.; ROY, P.S.; HARMSEN, K.; SAHA, S.K (ed.). Satellite Remote Sensing and GIS Applications in Agricultural Meteo- rology. AGM-8, WMO/TD-No. 1182. Dehra Dun: India, p. 235-261, 2004.

PAIXÃO, J.S.; ANDRADE, C.L.T.; GARCIA Y GARCIA, A.; AMARAL, T.A.; STEIDLE NETO, A.J.; MARIN, F.R. An Alternative Approach to the Actual Brazilian Maize Crop Zoning. Revista Brasileira de Milho e Sorgo, v. 13, n. 3, p. 347-363, 2014.

ROSSATO, L.; ALVALÁ, R.C.S.; MARENGO, J.A.; ZERI, M.; CUNHA, A.P.M.A.; PIRES, L.B.M.; BARBOSA, H.A. Impact of Soil Moisture on Crop Yields over Brazilian Semiarid. Frontiers in Environmental Science, v. 5, n. 73, p. 23-29, 2017.

SANS, L.M.A.; GUIMARÃES, D.P. Zoneamento Agrícola de Riscos Climáticos para a Cultura do Milho. Sete Lagoas: Embrapa Milho e Sorgo, 5 p., 2006.

SCARPARE, F.V. Simulação do Crescimento da Cana-deAçúcar pelo Modelo Agro-Hidrológico SWAP/ WOFOST. Tese de Doutorado em Física do Ambiente Agrícola, Escola Superior de Agricultura "Luiz de Queiróz"m 163 p.m 2011.

SILVA, F.D.S.; COSTA, R.L.; ANTONIO, M.A.V.; AFONSO, E.O.; SANTOS, D.M.; MATEUS, N.P.A.; ANTONIO, J.F. Tendências observadas da evapotranspiração potencial no estado de Alagoas (1961-2016). Revista Brasileira de Geografia Física, v. 11, n. 1, p. 28-43, 2018.

THORNTHWAITE, C.W.; MATHER, J.R. The Water Balance. Publications in Climatology, v. 8. Centerton: Drexel Institute of Technology, Laboratory of Climatology, 104 p., 1955.

VORPAGEL, A.G. Inoculação de Azospirillum, Isolado e Associado a Bioestimulante, em Milho, no Noroeste do RS. Trabalho de Conclusão de Curso, Universidade Regional do Noroeste do Estado do Rio Grande do Sul, 56 p., 2010.

XAVIER, A.C.; KING, C.W.; SCANLON, B.R. Daily gridded meteorological variables in Brazil (1980-2013). International Journal of Climatology, v. 36, n. 6, p. 2644-2659, 2016.

License information: This is an open-access article distributed under the terms of the Creative Commons Attribution License (type CC-BY), which permits unrestricted use, distribution and reproduction in any medium, provided the original article is properly cited. 